\title{
An Effective S-Box Construction Based on Linear Recurrences With Constant Co-Efficient
}

wajeeha Iftikhar ( $\nabla$ wajeehaiftikhar993@gmail.com )

University of Engineering and Technology Taxila, Pakistan

NASIR SIDDIQUI

University of Engineering and Technology Taxila, Pakistan

Muhammad Ehtisham ul Haq

University of Engineering and Technology Taxila, Pakistan

\section{Research Article}

Keywords: Substitution-box, Symmetric Cryptography, Linear Recurrence Formula, Image Encryption

Posted Date: March 16th, 2021

DOI: https://doi.org/10.21203/rs.3.rs-259399/v1

License: (1) This work is licensed under a Creative Commons Attribution 4.0 International License. Read Full License 


\title{
An effective S-box construction based on linear recurrences with constant co-efficient
}

Nasir Siddiqui ${ }^{a}$, Wajeeha Iftikhar ${ }^{a}$ and Muhammad Ehtisham ul Haq ${ }^{\mathrm{b}}$

\begin{abstract}
In this paper, we propose an algorithm for the construction of substitution-box through a linear recurrence relation. S-box is considered as the building block of block ciphers; here we present a technique to construct Substitution-boxes by using linear recurrence relation to comply with the standards of good quality S-boxes. We have witnessed that the latest research relies more on improving already existing techniques, rather than developing wholly new methods. We use linear recurrence in an entirely new way. Real initial and obtained values are concealed by converting it into new numbers to avoid any pattern to follow. The obtained S-boxes are analyzed through performance tests to check the strength and quality of our S-boxes. Later we will encrypt an image and also perform encryption and decryption in noise channels to make it more applicable in low profile applications.
\end{abstract}

Keywords Substitution-box, Symmetric Cryptography, Linear Recurrence Formula, Image Encryption

\section{Introduction}

The security of data has become a major necessity of our life in the present era. Fast and modern communication channels are being used around the globe, which demands the security of a transmitted data. In order to prevent the misuse of data by unauthorized people, many researchers are developing techniques to create confusion in the original data. As time progresses, the need

1

${ }^{\text {a }}$ Department of Basic Sciences, University of Engineering and Technology, Taxila, Pakistan

E-mail address: nasir.siddiqui@uettaxila.edu.pk (N. Siddiqui), wajeehaiftikhar993@gmail.com

(W. Iftikhar)

${ }^{\mathrm{b}}$ Department of Computer Engineering, University of Engineering and Technology, Taxila, Pakistan

E-mail address: ehatishamuet@gmail.com 
for cryptographically secure systems is becoming very important as we are using them not only in military applications, but also in our daily life in our mobile phones, social media accounts; Banks, remote control car lock systems [1].

Substitution-box plays a significant role in ensuring the security of the system and is considered to be a major component of a symmetric block ciphers. Substitution-box creates confusion in data by establishing a unique relationship between plaintext and cipher text. Most secure block ciphers include both substitution and permutation [2]. The purpose of this paper is to construct Sboxes, which satisfy all the necessary conditions of a secure system. It is a known fact that in the last two decades; the academic study of construction of Substitution-boxes for safety of our data has been an attractive area of study for many researchers. AES is considered as the strongest systems and is widely used, but due to the high computational complexities, comparatively easy applicable algorithms are developed these days[3].

The proposed technique of our S-box construction is based on linearly recurrence relation. The idea of using linear recurrence may be perceived as outdated in this era. However, we will use it in a unique way along with the latest methods to get efficient results.

In section 1, the preliminaries will be discussed. In section 2, the method for the construction of our substitution-box will be explained. In section 3, the comparison with already existing techniques will be discussed. The strength of the constructed S-boxes is tested on the basis of non-linearity, BIC (Bit Independence criterion, SAC (Strict Avalanche Criterion, LP (Linear Probability) and DP (Differential probability) [4], [5].

\section{Preliminaries}

\subsection{Linear recurrence formula:}

In order to incorporate linear recurrence in this research, I will have to delve into its prior history, which can be classified as finite history and full history [6]. Finite history emphasizes on the dependence of fixed number of earlier values while an equation that depends on all the preceding terms has a full history. Similar to differential equation it can be classified into homogeneous and non-homogenous. Homogenous linear recurrence relations are easily distinguishable from non-homogenous relations due to the presence of an extra term $g(x)$.The formula of linear recurrence is defined in equation (1).

$$
c_{0} x_{n}+c_{1} x_{n-1}+\cdots+c_{m} x_{n-m}=g(x), \quad n \geq m
$$


If $g(x)=0$,equation is homogenous, but when it is not equal to zero, then equation is nonhomogenous. Here we have considered a homogenous equation with all coefficients zero except $c_{0}, c_{1}$ and $c_{2}$. Here we take the values as $c_{0}=1, c_{1}=-1$ and $c_{2}=-1$.

\section{Scheme of Proposed S-box:}

\subsection{Step 1}

First we consider the Fibonacci number calculation formula [6]. We are using the following equation.

$$
x_{n}=x_{n-1}+x_{n-2}
$$

Consider two initial values randomly.

\subsection{Step 2:}

The values are calculated. But after many iterations maximum of 168 values are calculated. To calculate more values we apply the same formula with slight change in initial conditions. The process repeats until we get all required values. We get all values by using a formula three to four times with the change in initial conditions and using different variables. For example this time we use equation (3).

$$
y_{n}=y_{n-1}+y_{n-2}
$$

We can also change the formula as a product of preceding terms instead of summation to create more confusion.

\subsection{Step 3:}

The numbers are arranged in specific way that calculated values are picked in iterations in a respective manner. For example, the first value from $\mathrm{x}$ is used, then the first value of $\mathrm{y}$ and so on. This step will make all values more random. The program is designed in MATLAB software.

Table 1 Initial values

\begin{tabular}{cccccccc}
\hline $\boldsymbol{x}_{\mathbf{0}}$ & $\boldsymbol{x}_{\mathbf{1}}$ & $\boldsymbol{y}_{\mathbf{0}}$ & $\boldsymbol{y}_{\mathbf{1}}$ & $\boldsymbol{z}_{\mathbf{0}}$ & $\boldsymbol{z}_{\mathbf{1}}$ & $\boldsymbol{t}_{\mathbf{0}}$ & $\boldsymbol{t}_{\mathbf{1}}$ \\
\hline $\mathbf{1 5 0}$ & 130 & 185 & 146 & 180 & 165 & 95 & 240 \\
\hline
\end{tabular}




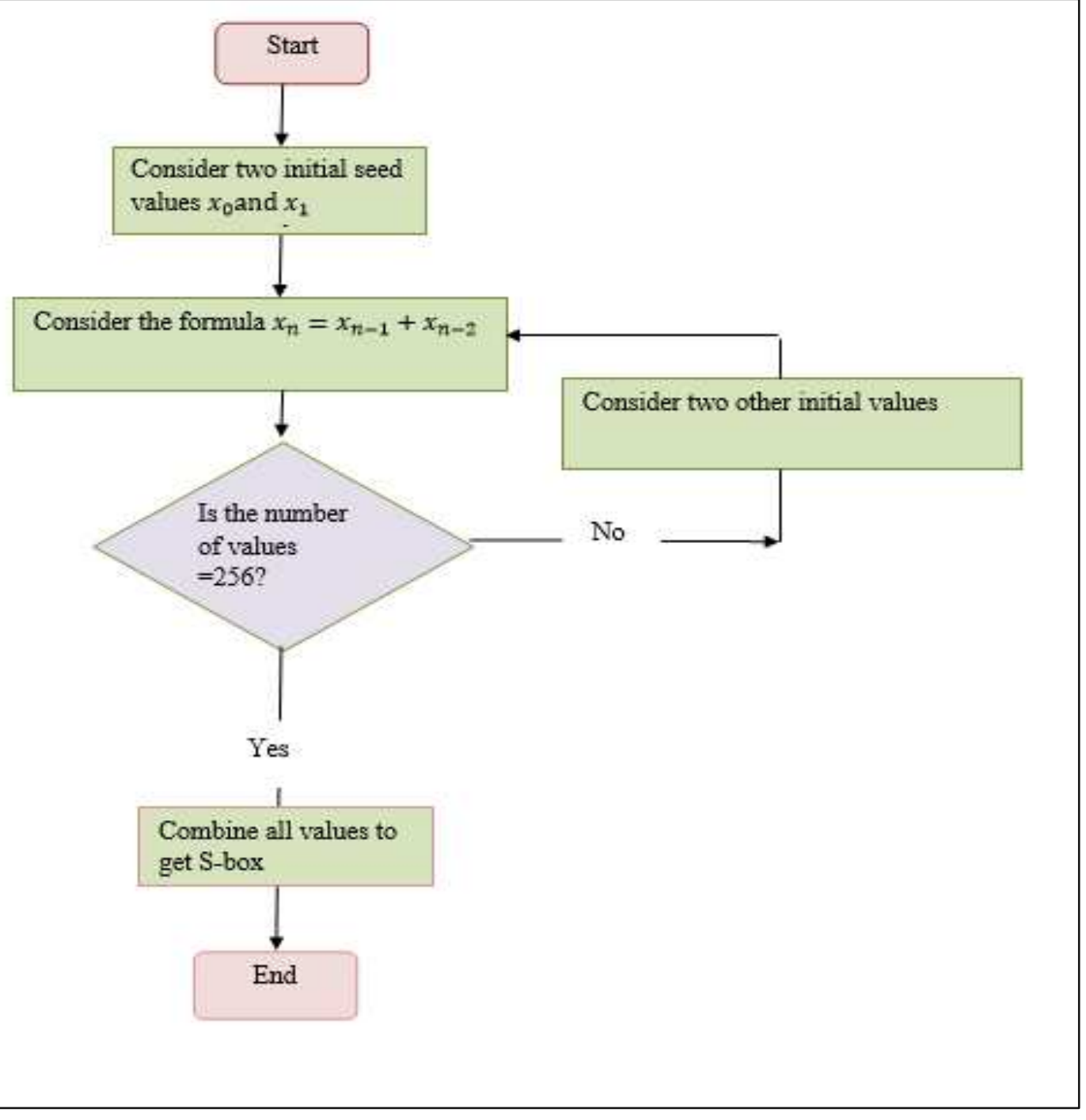

f ig 1 Flowchart Representation 
The S-box obtained by following these steps is shown in table 2.All the values are obtained with $\mathrm{x}, \mathrm{y}$ and $\mathrm{z}$ variables. But to create more confusion, in the last step we are using formula presented in equation (4)

$$
t_{n}=t_{n-1} \times t_{n-2}
$$

Table 2 Proposed S-box after three steps

\begin{tabular}{|c|c|c|c|c|c|c|c|c|c|c|c|c|c|c|c|}
\hline 150 & 185 & 180 & 95 & 130 & 146 & 165 & 240 & 24 & 75 & 89 & 16 & 154 & 221 & 254 & 0 \\
\hline 178 & 40 & 87 & 76 & 5 & 85 & 45 & 172 & 74 & 50 & 1 & 72 & 173 & 145 & 174 & 218 \\
\hline 91 & 108 & 129 & 9 & 70 & 113 & 100 & 242 & 109 & 248 & 99 & 209 & 170 & 62 & 162 & 184 \\
\hline 15 & 13 & 77 & 238 & 197 & 92 & 58 & 210 & 169 & 151 & 98 & 105 & 138 & 179 & 236 & 97 \\
\hline 118 & 20 & 117 & 216 & 59 & 137 & 18 & 135 & 133 & 94 & 157 & 12 & 8 & 143 & 46 & 186 \\
\hline 203 & 249 & 38 & 161 & 196 & 189 & 211 & 2 & 191 & 253 & 78 & 181 & 188 & 232 & 71 & 217 \\
\hline 106 & 32 & 163 & 86 & 25 & 116 & 66 & 69 & 152 & 43 & 61 & 114 & 104 & 183 & 190 & 10 \\
\hline 33 & 200 & 141 & 48 & 233 & 6 & 36 & 120 & 67 & 49 & 234 & 111 & 28 & 250 & 82 & 201 \\
\hline 168 & 247 & 229 & 73 & 64 & 147 & 65 & 54 & 212 & 34 & 21 & 88 & 27 & 122 & 136 & 231 \\
\hline 245 & 30 & 125 & 204 & 177 & 239 & 80 & 171 & 230 & 132 & 93 & 56 & 225 & 101 & 194 & 31 \\
\hline 14 & 149 & 124 & 90 & 167 & 42 & 96 & 131 & 22 & 121 & 52 & 11 & 26 & 23 & 126 & 237 \\
\hline 44 & 202 & 81 & 112 & 193 & 198 & 228 & 35 & 207 & 110 & 220 & 226 & 41 & 128 & 115 & 246 \\
\hline 148 & 251 & 205 & 222 & 140 & 79 & 144 & 139 & 166 & 68 & 127 & 29 & 206 & 60 & 7 & 53 \\
\hline 153 & 160 & 57 & 214 & 244 & 235 & 119 & 176 & 134 & 164 & 3 & 241 & 47 & 156 & 192 & 83 \\
\hline 182 & 84 & 219 & 158 & 175 & 208 & 107 & 102 & 4 & 37 & 223 & 142 & 252 & 103 & 224 & 215 \\
\hline 213 & 17 & 227 & 51 & 187 & 63 & 199 & 55 & 195 & 19 & 155 & 159 & 39 & 243 & 123 & 255 \\
\hline
\end{tabular}

It can be observed that the all our initial values are appearing in the first row, so overcome this problem we will have to move on to the next step.

\subsection{Step 4}

The actual obtained S-box is transformed by converting the values from decimal to binary and permuted in reverse order to get the new decimal numbers, which does not affect the nonlinearity of the S-box; rather it helps us hide the initial values. 


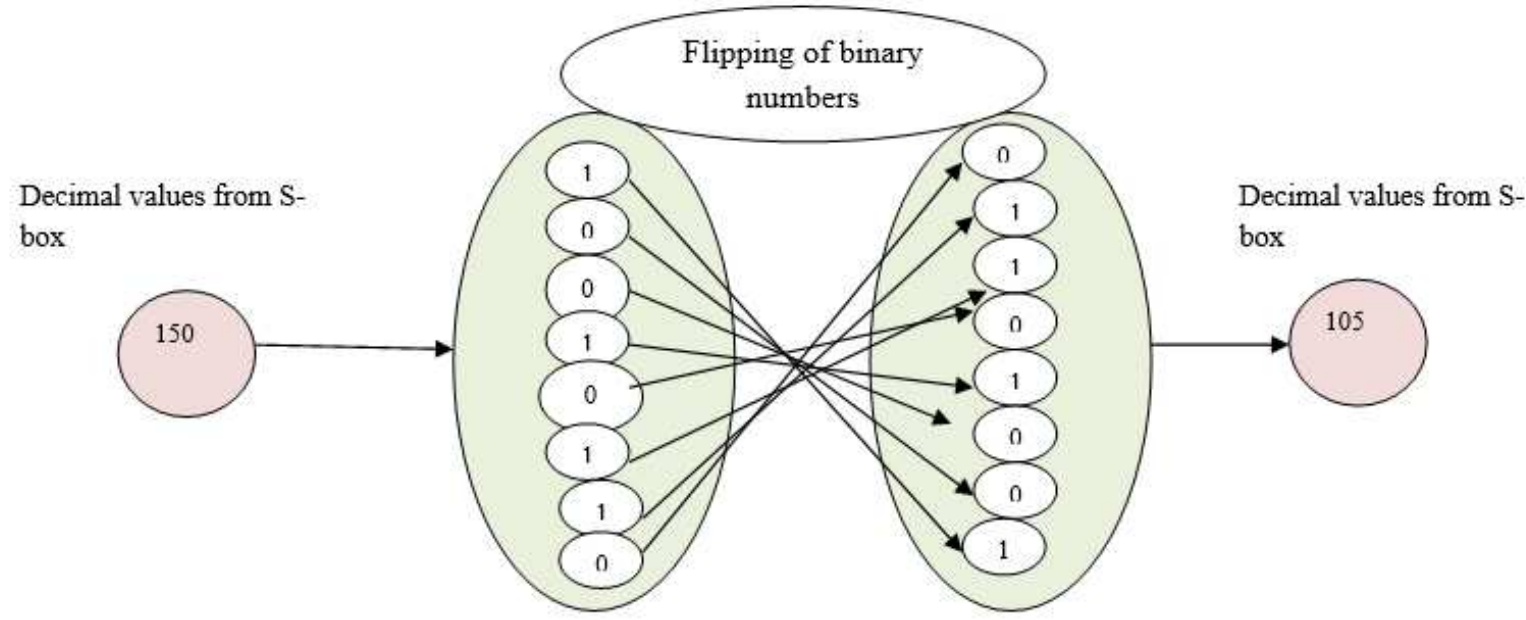

fig 2 Flipping of numbers

Similar process is applied to every value in the S-box to transform our S-box into a new S-box.

Table 3 Final Proposed S-box

\begin{tabular}{|c|c|c|c|c|c|c|c|c|c|c|c|c|c|c|c|}
\hline 105 & 157 & 45 & 250 & 65 & 73 & 165 & 15 & 24 & 210 & 154 & 8 & 89 & 187 & 127 & 0 \\
\hline 77 & 20 & 234 & 50 & 160 & 170 & 180 & 53 & 82 & 76 & 128 & 18 & 181 & 137 & 117 & 91 \\
\hline 218 & 54 & 129 & 144 & 98 & 142 & 38 & 79 & 182 & 31 & 198 & 139 & 85 & 124 & 69 & 29 \\
\hline 240 & 176 & 178 & 119 & 163 & 58 & 92 & 75 & 149 & 233 & 70 & 150 & 81 & 205 & 55 & 134 \\
\hline 110 & 40 & 174 & 27 & 220 & 145 & 72 & 225 & 161 & 122 & 185 & 48 & 16 & 241 & 116 & 93 \\
\hline 211 & 159 & 100 & 133 & 35 & 189 & 203 & 64 & 253 & 191 & 114 & 173 & 61 & 23 & 226 & 155 \\
\hline 86 & 4 & 197 & 106 & 152 & 46 & 66 & 162 & 25 & 212 & 188 & 78 & 22 & 237 & 125 & 80 \\
\hline 132 & 19 & 177 & 12 & 151 & 96 & 36 & 30 & 194 & 140 & 87 & 246 & 56 & 95 & 74 & 147 \\
\hline 21 & 239 & 167 & 146 & 2 & 201 & 130 & 108 & 43 & 68 & 168 & 26 & 216 & 94 & 17 & 231 \\
\hline 175 & 120 & 190 & 51 & 141 & 247 & 10 & 213 & 103 & 33 & 186 & 28 & 135 & 166 & 67 & 248 \\
\hline 112 & 169 & 62 & 90 & 229 & 84 & 6 & 193 & 104 & 158 & 44 & 208 & 88 & 232 & 126 & 183 \\
\hline 52 & 83 & 138 & 14 & 131 & 99 & 39 & 196 & 243 & 118 & 59 & 71 & 148 & 1 & 206 & 111 \\
\hline 41 & 223 & 179 & 123 & 49 & 242 & 9 & 209 & 101 & 34 & 254 & 184 & 115 & 60 & 224 & 172 \\
\hline 153 & 5 & 156 & 107 & 47 & 215 & 238 & 13 & 97 & 37 & 192 & 143 & 244 & 57 & 3 & 202 \\
\hline 109 & 42 & 219 & 121 & 245 & 11 & 214 & 102 & 32 & 164 & 251 & 113 & 63 & 230 & 7 & 235 \\
\hline 171 & 136 & 199 & 204 & 221 & 252 & 227 & 236 & 195 & 200 & 217 & 249 & 228 & 207 & 222 & 255 \\
\hline
\end{tabular}

\section{Analysis criterion of S-boxes:}

The In order to investigate the strength of our constructed S-box, we will have to test our substitution box according to the following criterions such as Non-Linearity, Strict Avalanche Criterion, Linear Probability, Differential probability and bit independence criterion as explained in [7], [8]. The values obtained are presented in the Table 4. 
Table 4 Analysis of the constructed S-box

\begin{tabular}{|l|l|l|l|l|l|l|}
\hline Analysis & Max Value & Min Value & $\begin{array}{l}\text { Average } \\
\text { value }\end{array}$ & LP & DP & $\begin{array}{l}\text { Square } \\
\text { deviation }\end{array}$ \\
\hline $\begin{array}{l}\text { Non } \\
\text { linearity }\end{array}$ & 108 & 104 & 106 & & & \\
\hline SAC & 0.609375 & 0.406250 & 0.497070 & & & 0.046408 \\
\hline LP & 160.0 & & & 0.125 & & \\
\hline DP & & & & & 0.039062 & \\
\hline BIC & 103.571 & 98.000 & 2.1 & & & \\
\hline
\end{tabular}

Table 5 Comparisons with different S-boxes

\begin{tabular}{|c|c|c|c|c|c|c|c|}
\hline \multirow{2}{*}{ S-boxes } & \multicolumn{3}{|c|}{ Non- linearity } & \multirow[t]{2}{*}{ SAC } & \multirow[t]{2}{*}{ LP } & \multirow[t]{2}{*}{ DP } & \multirow[t]{2}{*}{ BIC } \\
\hline & Maximum & Minimum & $\begin{array}{l}\text { Average } \\
\text { Value }\end{array}$ & & & & \\
\hline Proposed S-box & 108 & 104 & 106 & 0.4970 & 160 & 0.039 & 103.57 \\
\hline AES S-box[9] & 112 & 112 & 112 & 0.504 & 144 & 0.0156 & 112 \\
\hline APA S-box[10] & 112 & 112 & 112 & 0.5 & 144 & 0.0156 & 112 \\
\hline Gray S-box[11] & 112 & 112 & 112 & 0.499 & 144 & 0.0156 & 112 \\
\hline$S_{8}$ AES S-box[12] & 112 & 112 & 112 & 0.504 & 144 & 0.0156 & 112 \\
\hline Skipjack S-box[13] & 108 & 104 & 105.75 & 0.503 & 156 & 0.0468 & 104.14 \\
\hline Xyi S-box[14] & 106 & 104 & 105 & 0.502 & 168 & 0.0468 & 103.78 \\
\hline $\begin{array}{l}\text { Residue } \\
\text { prime[15],[16] }\end{array}$ & 104 & 94 & 99.5 & 0.516 & 162 & 0.281 & 101.71 \\
\hline [17] & 108 & 102 & 104.5 & 0.4980 & 160 & 0.0469 & 104.64 \\
\hline
\end{tabular}

\subsection{Non-linearity:}

S-box is designed in such a way that the relation between plaintext and cipher text is nonlinear in terms of mapping, otherwise it would be susceptible to linear cryptanalysis. Non-linearity is actually the distance between a Boolean function and the set of all the affine functions. The importance of non-linearity for the encryption process cannot be denied [8].

The calculated non-linearity of our S-box is 106, with minimum non-linearity as 104 and maximum non-linearity as 108.The comparison with the non-linearity of the known ciphers is presented in the table 6 and Figure 3. 
Table 6 Comparison of Non-Linearity of existing S-boxes

\begin{tabular}{|c|c|c|c|c|c|c|c|c|c|}
\hline S-boxes & $\mathbf{0}$ & 1 & 2 & 3 & 4 & 5 & 6 & 7 & $\begin{array}{l}\text { Average } \\
\text { Non- } \\
\text { linearity }\end{array}$ \\
\hline Proposed S-box & 104 & 106 & 106 & 104 & 108 & 106 & 108 & 106 & 106 \\
\hline AES S-box[9] & 112 & 112 & 112 & 112 & 112 & 112 & 112 & 112 & 112 \\
\hline APA S-box $[10]$ & 112 & 112 & 112 & 112 & 112 & 112 & 112 & 112 & 112 \\
\hline Gray S-box[11] & 112 & 112 & 112 & 112 & 112 & 112 & 112 & 112 & 112 \\
\hline $\begin{array}{l}S_{8} \text { AES } \\
\text { box }[12]\end{array}$ & 112 & 112 & 112 & 112 & 112 & 112 & 112 & 112 & 112 \\
\hline $\begin{array}{l}\text { Skipjack } \\
\text { box }[13]\end{array}$ & 104 & 104 & 108 & 108 & 108 & 104 & 104 & 106 & 105.75 \\
\hline Xyi S-box[14] & 106 & 104 & 104 & 106 & 104 & 106 & 104 & 106 & 105 \\
\hline $\begin{array}{l}\text { Residue } \\
\text { prime[15],[16] }\end{array}$ & 94 & 100 & 104 & 104 & 102 & 100 & 98 & 94 & 99.5 \\
\hline
\end{tabular}

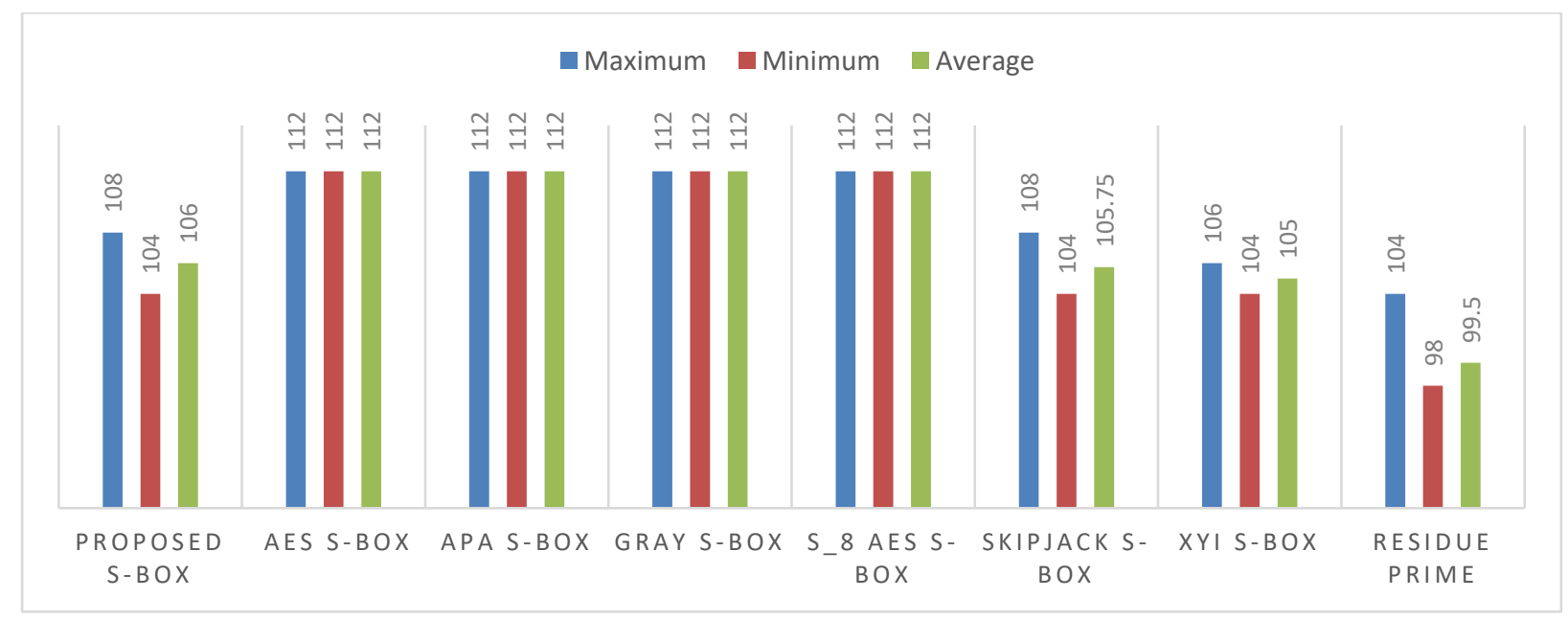

fig 3 Non linearity

\subsection{Strict Avalanche Criterion:}

According to the Strict Avalanche Criterion, if in the function that is forming the substitution box we change the single bit of input it is necessary that there is probability of $50 \%$ change in the output bits sequence. If the value is closer to AES, it becomes more reliable. The obtained strict avalanche value for our constructed S-Box is better than skipjack s-box and residue prime. 


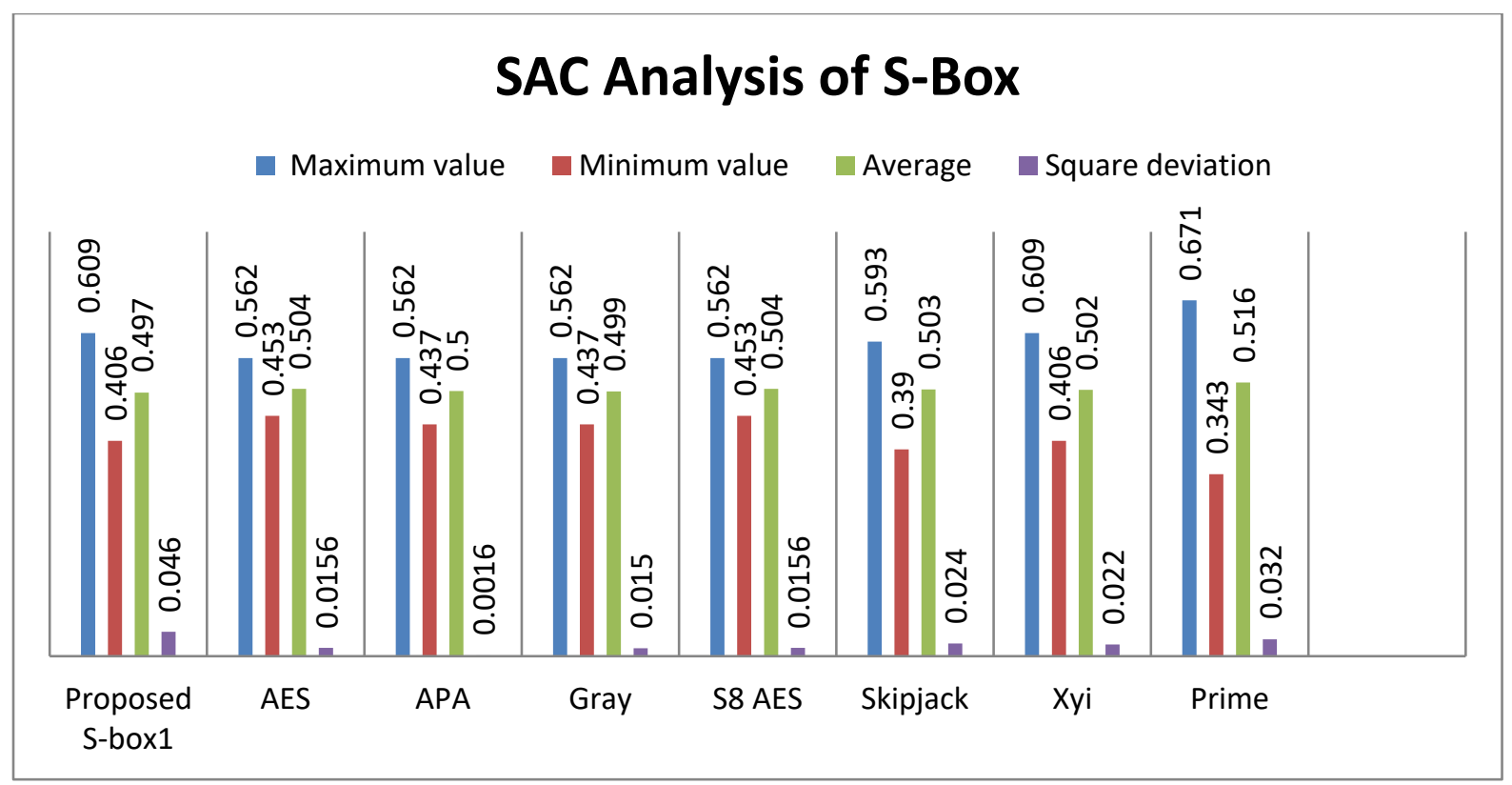

fig 4 Strict Avalanche criterions

\subsection{Bit Independence Criterion:}

The independent behavior of the pair of variables and the changes in input bits are considered as significant factors of bit independence criterion. According to this criterion, input bits are transformed completely, and then output bits are checked for their independence. Bit independence is one of the most important criterions of S-box strength.

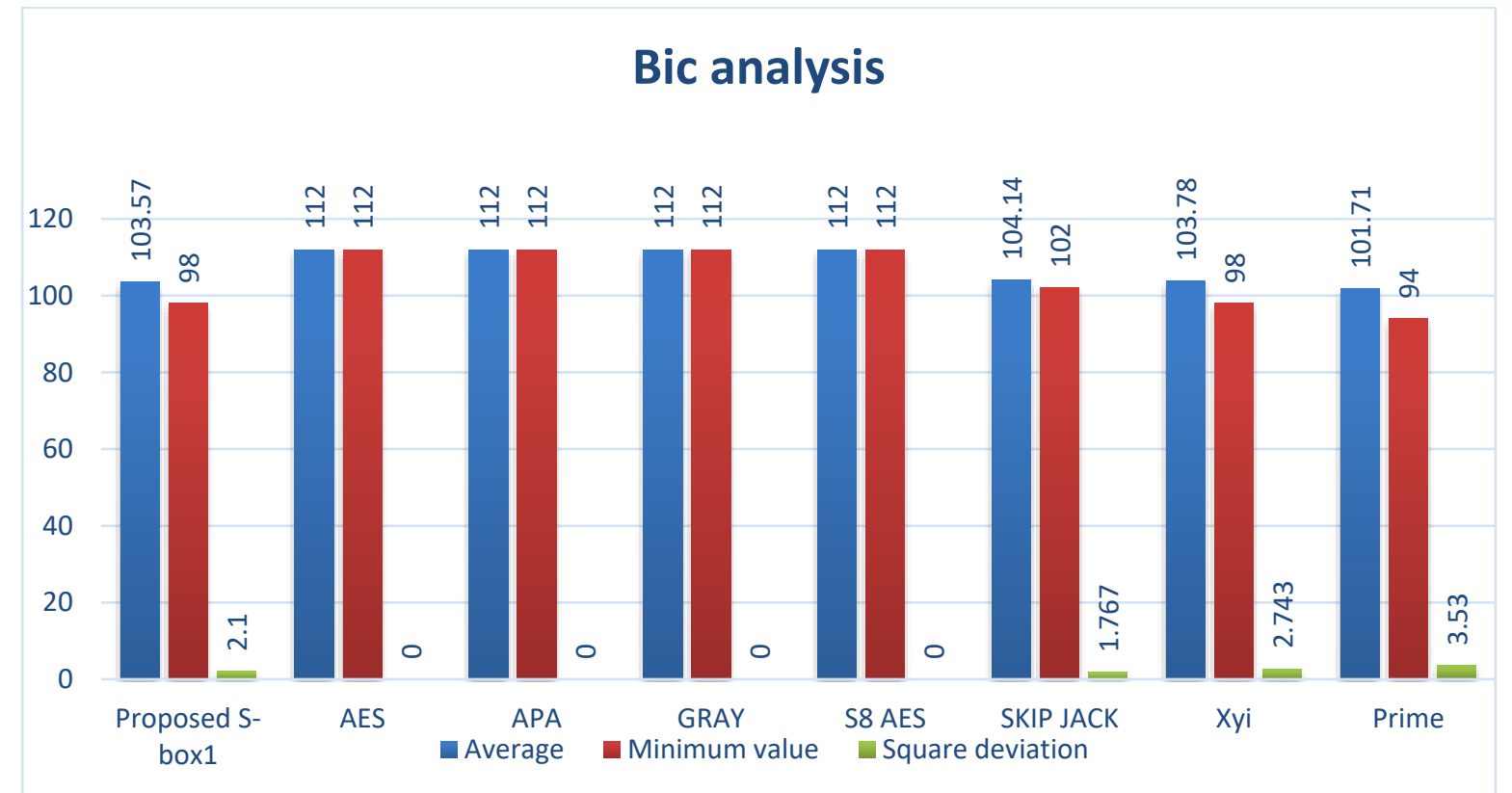

fig 5 Bit Independence Criterion 


\subsection{Differential probability and Linear Probability}

To access the imbalance of an event, the linear probability of the constructed S-box is calculated. It is represented by the formula given in equation (5)

$$
\mathrm{LP}=\max _{\beta \mathrm{p}, \beta \mathrm{q} \neq 0}\left|\frac{\#\left\{x \varepsilon G F\left(2^{8}\right) / x \cdot \beta p=s(x) \cdot \beta q\right\}}{2^{q}}-\frac{1}{2}\right|
$$

In the given formula, $p$ represents the input values of S-box. The input and output masks are expressed in the form of $\beta p, \beta q$ respectively. We have compared our results with standard results. Our obtained value is 0.125 , which is not too susceptible in terms of linear attacks [18].

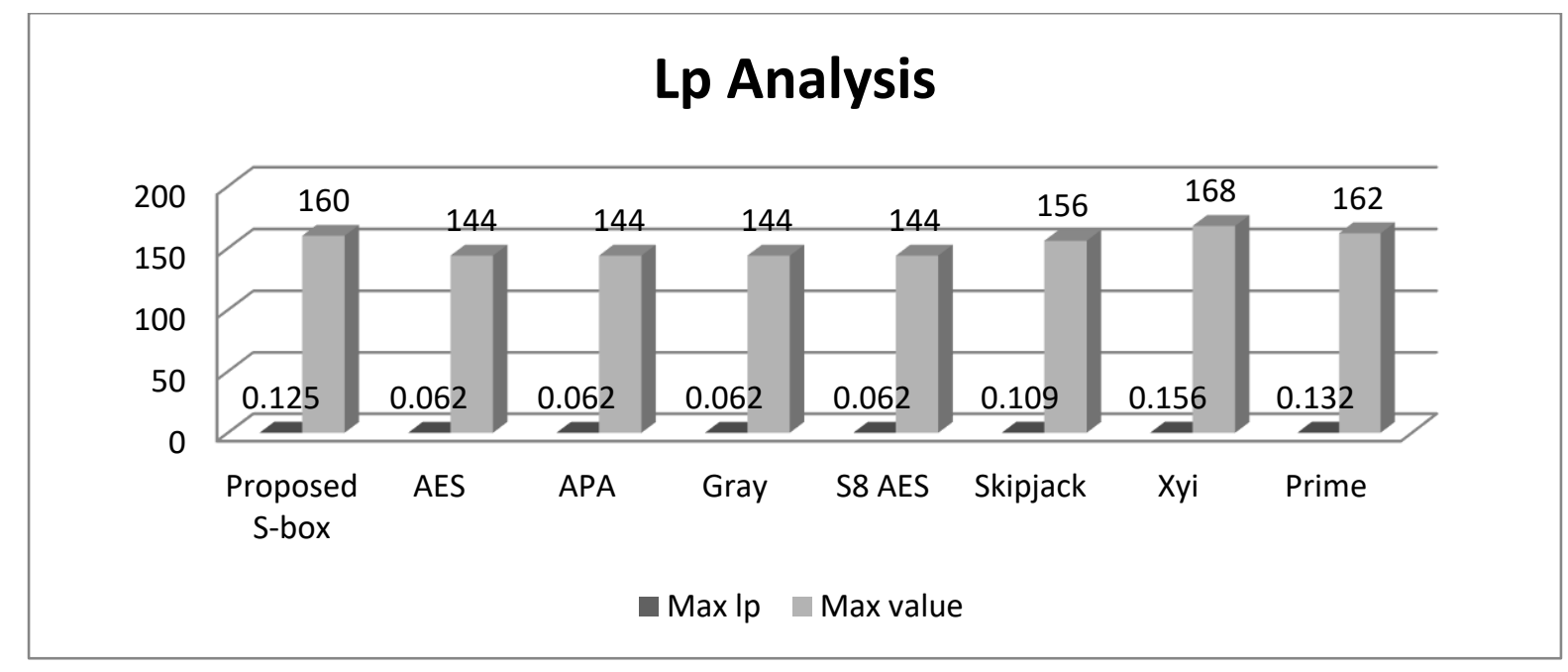

fig 6 Linear Probability

To measure the differential uniformity of the constructed S-box we will check the differential probability of the constructed S-box. The formula for the calculation of differential probability is expressed in the following form in equation (6)

$$
D P=\left[\frac{\#\{x \in X / S(x) \oplus S(y \oplus \Delta x=\Delta y\}}{2^{p}}\right]
$$

To represent the differentials we will use $\Delta x$ and $\Delta y$. On the basis on comparative analysis, we can say that our results are better than Skipjack, $X_{y i}$ and residue prime [18]. 


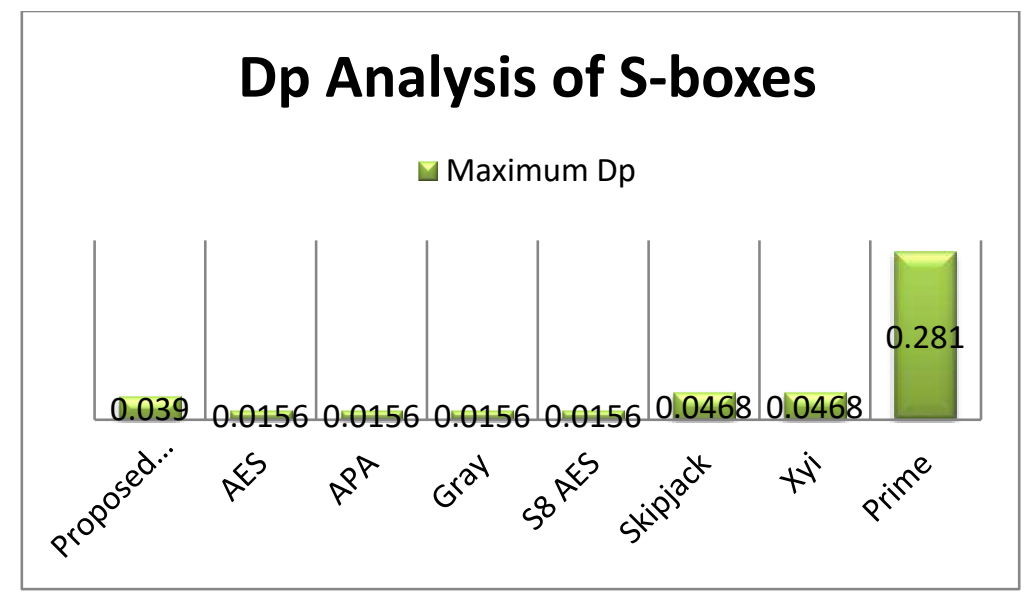

fig 7 Differential probability

\section{Image Encryption:}

Here we encrypt the image in order to test the strength of the constructed S-box. We are using capsicum image for the encryption process. The image from pixel value of 0 to 266 is considered for encryption and the S-box ranging from 0 to 256 constructed is applied on the image, which will replace values of the image according to the our S-box. The algorithm is applied twice to get the best encrypted image.

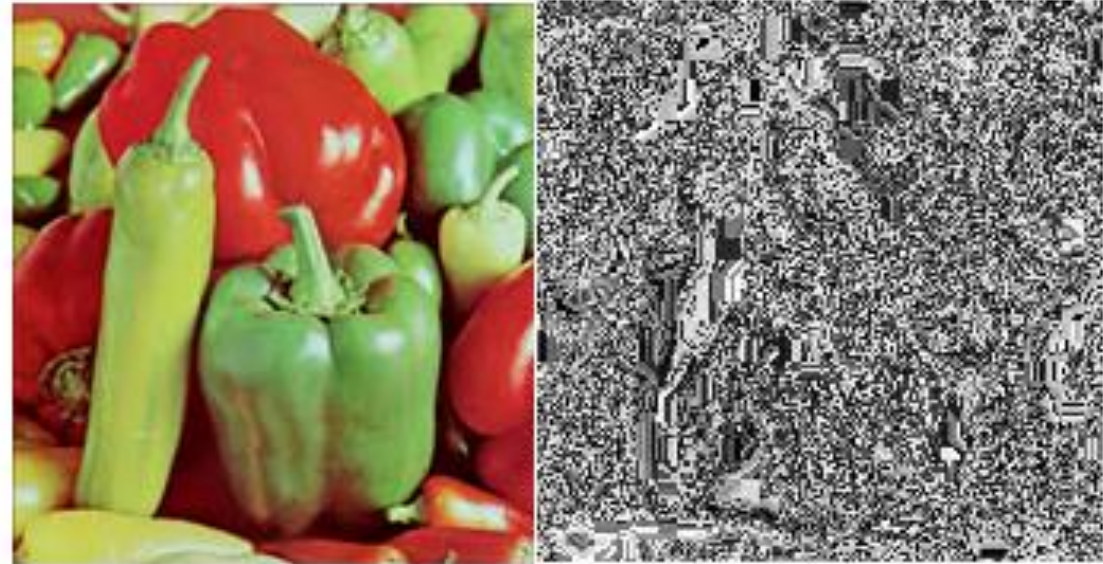

(a) (b)

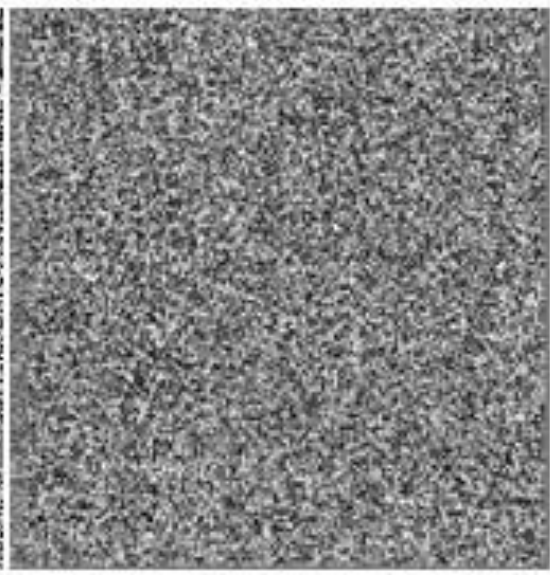

(c)

fig 8 Capsicum Image Encryption (a) Original Image (b) First Round Encrypted Image and(c) Final Encrypted image

Table 7 Image encryption using first algorithm

\begin{tabular}{lllccc}
\hline Image & Entropy & $\begin{array}{l}\text { Correlation } \\
\text { coefficient }\end{array}$ & Energy & Contrast & Homogeneity \\
\hline Pepper Image & 7.5846 & 0.1013 & 0.0178 & 9.2410 & 0.4663 \\
\hline
\end{tabular}




\section{Noise resistant property of the proposed Encryption Scheme:}

As we know, that the intruder can add noise to any communication channel intentionally. This makes it necessary to develop an algorithm, which is noise resistant. The noise must be removed to reduce the effect of channel noise caused by unauthorized person [20], [21].

The next step is followed by the analysis of our constructed S-box on the basis of bit error rate (BER) which is actually a function of length of burst errors for multiple values of SNR in aggregation with the single error correction code. We will have to compare the performance of our constructed S-box with conventional random S-box, Algebraic S-box and Chaotic S-Box. The conventional S-box is available in MATLAB as RANDINTRLV function, which makes MATLAB software to be a necessary tool. The data to taken is in the 100 random blocks with the size $\mathrm{N}=256$. To encode our data we are using linear hamming code. The length of the cord word is $(7,4)$ whereas the minimum value of the hamming distance will be 3 in order to rectify even a single error. WE will have to introduce the burst of errors with different lengths and then we will XOR it with modular data to analyze the performance against burst errors. Error rates are calculated and compared with the standard S-boxes error rates at different values of SNR. We will compare our results with the AES, Gray, Skipjack and Residue prime S-boxes. The values for known S-boxes are taken from research paper [22].

Table 8 SNR=5 Error rate

\begin{tabular}{|l|l|l|l|l|l|l|}
\hline $\begin{array}{l}\text { Proposed } \\
\text { box }\end{array}$ & 0.48836 & 0.52672 & 0.51432 & 0.49176 & 0.50002 & 0.51988 \\
\hline AES & 0.51024 & 0.49726 & 0.50752 & 0.48836 & 0.50478 & 0.50342 \\
\hline Gray & 0.50068 & 0.4726 & 0.49452 & 0.52324 & 0.49314 & 0.48764 \\
\hline skipjack & 0.487 & 0.4997 & 0.4910 & 0.5232 & 0.4917 & 0.4883 \\
\hline residue & 0.50134 & 0.50684 & 0.51574 & 0.4863 & 0.51988 & 0.51438 \\
\hline
\end{tabular}

Table 9 SNR=10 Error rate

\begin{tabular}{|l|l|l|l|l|l|l|}
\hline $\begin{array}{l}\text { Proposed } \\
\text { box }\end{array}$ & 0.41966 & 0.4175 & 0.42868 & 0.4328 & 0.40998 & 0.44062 \\
\hline AES & 0.42626 & 0.41104 & 0.42532 & 0.42826 & 0.41532 & 0.42396 \\
\hline Gray & 0.41404 & 0.42832 & 0.4167 & 0.42144 & 0.41014 & 0.43382 \\
\hline skipjack & 0.4184 & 0.4085 & 0.4079 & 0.429 & 0.4137 & 0.4226 \\
\hline residue & 0.43694 & 0.4143 & 0.42026 & 0.41984 & 0.41216 & 0.43372 \\
\hline
\end{tabular}


Table 10 SNR=15 Error rate

\begin{tabular}{|l|l|l|l|l|l|l|}
\hline $\begin{array}{l}\text { Proposed } \\
\text { box }\end{array}$ & 0.00668 & 0.01052 & 0.01866 & 0.02438 & 0.03308 & 0.04632 \\
\hline AES & 0.00618 & 0.01294 & 0.02292 & 0.02988 & 0.03636 & 0.04592 \\
\hline Gray & 0.00606 & 0.0125 & 0.0164 & 0.02808 & 0.03486 & 0.05184 \\
\hline skipjack & 0.0183 & 0.0306 & 0.0389 & 0.0540 & 0.0562 & 0.0635 \\
\hline residue & 0.0060 & 0.01578 & 0.02082 & 0.0273 & 0.03064 & 0.0379 \\
\hline
\end{tabular}

\section{Conclusion}

In this work, we proposed a new scheme for the construction of S-boxes. We have established that linear recurrence relation can be used to construct efficient S-boxes in a simpler way. The technique presented for construction has better than some known algorithms. The strength of constructed S-boxes is analyzed by performance tests in order to cope up to the standards of good S-boxes. Furthermore, the constructed S-box is used for the encryption of an image and noise removal.

\section{References:}

[1] Paar, C., \& Pelzl, J. (2009). Understanding cryptography: a textbook for students and practitioners. Springer Science \& Business Media.

[2] Greene, D. H., \& Knuth, D. E. (2007). Mathematics for the Analysis of Algorithms. Springer Science \& Business Media

[3] Sarfraz, M., Hussain, I., Ali, F., \& Rasheed, A. (2016). A Mobius Transformation Based Algorithm for the Construction of Cryptographically Strong 131028 S-Boxes Having Highly Nonlinear. International Journal of Computer Science and Information Security, 14(5), 611.

[4] Ahmad, M., Haleem, H., \& Khan, P. M. (2014, February). A new chaotic substitution box design for block ciphers. In 2014 International Conference on Signal Processing and Integrated Networks (SPIN) (pp. 255-258). IEEE.

[5] Han, J. S., Kim, H. S., \& Neggers, J. (2012). On Fibonacci functions with Fibonacci numbers. Advances in Difference Equations, 2012(1), 126.

[6] Wilf, H. S. (2005). generatingfunctionology. CRC press.

[7] Zahid, A. H., \& Arshad, M. J. (2019). An Innovative Design of Substitution-Boxes Using Cubic Polynomial Mapping. Symmetry, 11(3), 437.

[8] Farwa, S., Muhammad, N., Shah, T., \& Ahmad, S. (2017). A novel image encryption based on algebraic S-box and Arnold transform. 3D Research, 8(3), 26.

[9] Daemen, J., \& Rijmen, V. (2013). The design of Rijndael: AES-the advanced encryption standard. Springer Science \& Business Media.

[10] Cui, L., \& Cao, Y. (2007). A new S-box structure named Affine-PowerAffine. International Journal of Innovative Computing, Information and Control, 3(3), 751-759. 
[11] Tran, M. T., Bui, D. K., \& Duong, A. D. (2008, December). Gray S-box for advanced encryption standard. In 2008 International Conference on Computational Intelligence and Security (Vol. 1, pp. 253-258). IEEE.

[12] Hussain, I., Shah, T., \& Mahmood, H. (2010). A new algorithm to construct secure keys for AES. International Journal of Contemporary Mathematical Sciences, 5(26), 12631270.

[13] Hussain, I., Shah, T., Gondal, M. A., \& Wang, Y. (2011). Analyses of SKIPJACK Sbox. World Appl. Sci. J, 13(11), 2385-2388.

[14] Hussain, I., Shah, T., Gondal, M. A., Khan, W. A., \& Mahmood, H. (2013). A group theoretic approach to construct cryptographically strong substitution boxes. Neural Computing and Applications, 23(1), 97-104.

[15] Abuelyamam, E. S. (2013, August). Residues of prime numbers as entries for the SBox. In 2013 INTERNATIONAL CONFERENCE ON COMPUTING, ELECTRICAL AND ELECTRONIC ENGINEERING (ICCEEE) (pp. 584-588). IEEE.

[16] Hussain, I., Shah, T., Mahmood, H., Gondal, M. A., \& Bhatti, U. Y. (2011). Some analysis of S-box based on residue of prime number. Proc Pak Acad Sci, 48(2), 111115.

[17] Liu, L., Zhang, Y., \& Wang, X. (2018). A novel method for constructing the S-box based on spatiotemporal chaotic dynamics. Applied Sciences, 8(12), 2650.

[18] Khan, M., \& Shah, T. (2015). An efficient construction of substitution box with fractional chaotic system. Signal, Image and Video Processing, 9(6), 1335-1338.

[19] Cheung, J. M. (2010). The design of S-boxes (Doctoral dissertation, Sciences).

[20] Hussain, I., Anees, A., Aslam, M., Ahmed, R., \& Siddiqui, N. (2018). A noise resistant symmetric key cryptosystem based on S 8 S-boxes and chaotic maps. The European Physical Journal Plus, 133(4), 167.

[21] Hussain, I., Shah, T., Gondal, M. A., \& Khan, W. A. (2011). Construction of cryptographically strong $8 \times 8$ S-boxes. World Applied Sciences Journal, 13(11), 23892395.

[22] Siddiqui, N., Naseer, A., \& Ehatisham-ul-Haq, M. (2020). A Novel Scheme of Substitution-Box Design Based on Modified Pascal's Triangle and Elliptic Curve. Wireless Personal Communications, 1-16. 
Figures

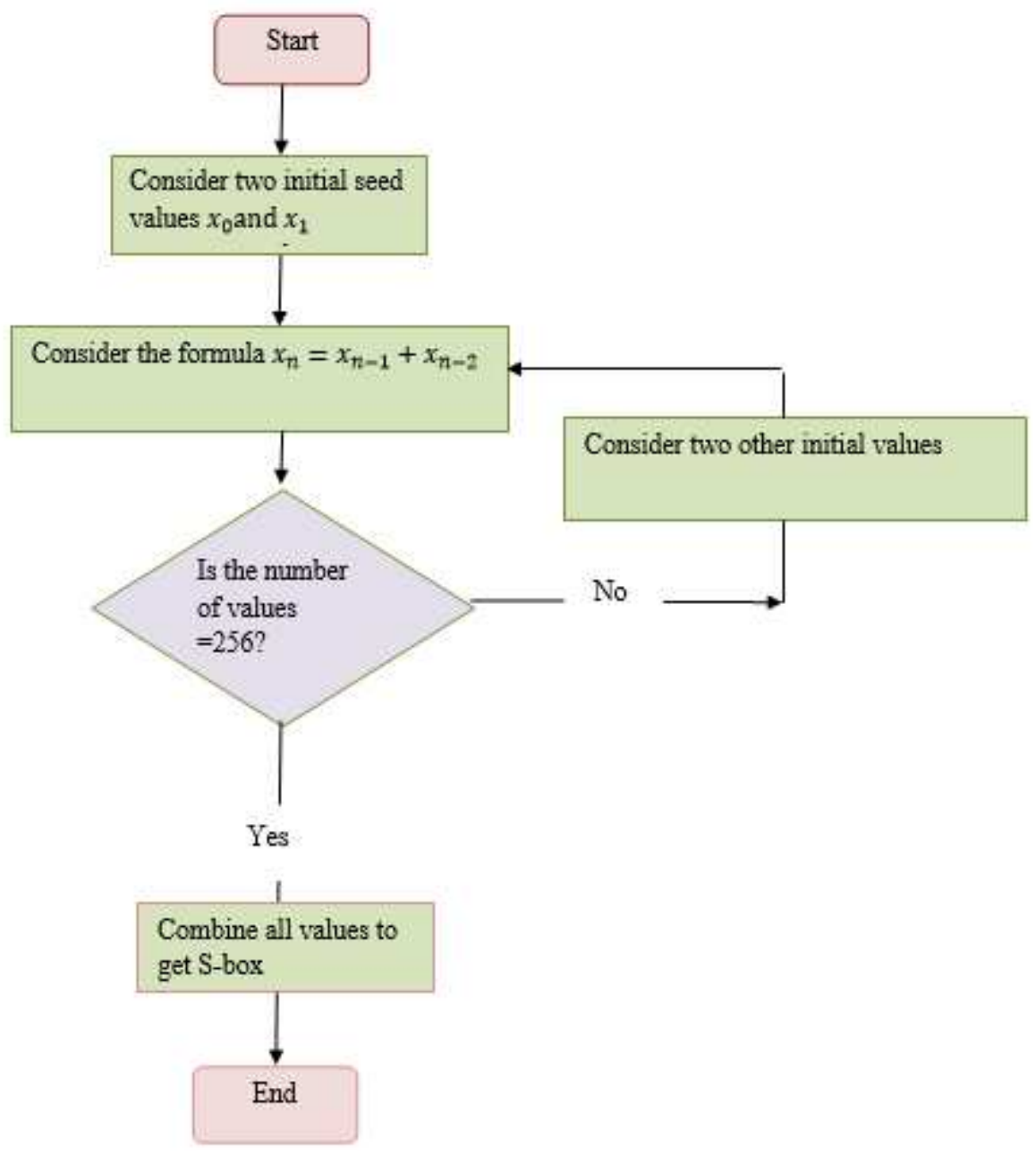

Figure 1

Flowchart Representation 


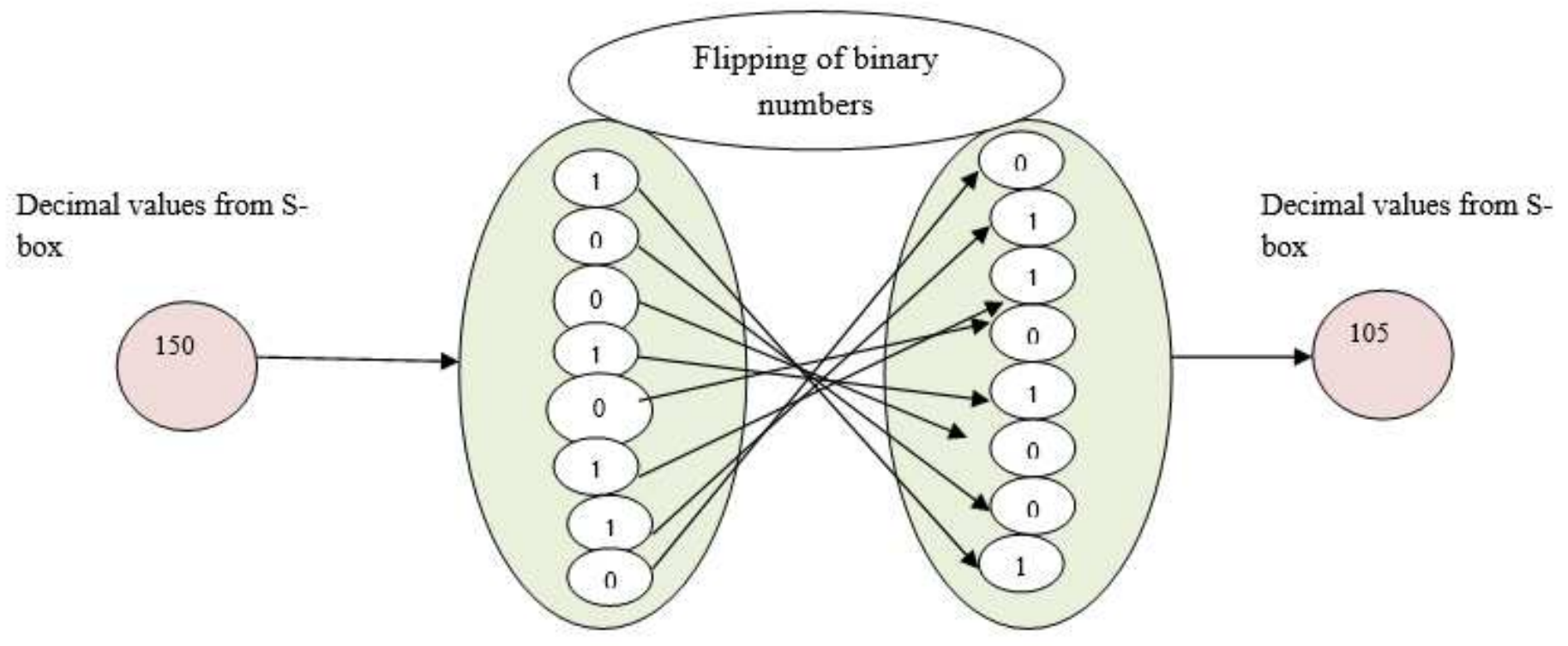

Figure 2

Flipping of numbers

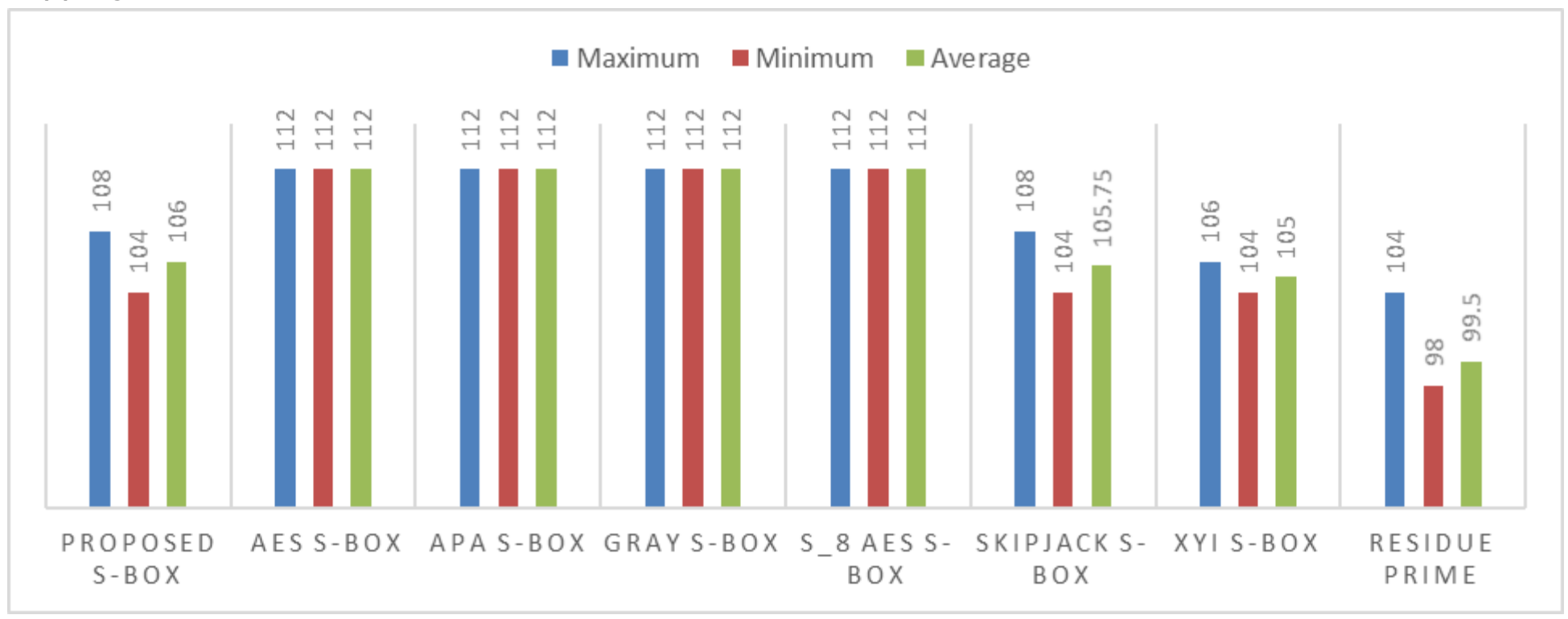

Figure 3

Non linearity 


\section{SAC Analysis of S-Box}

घaximum value $\quad$ Minimum value $\quad$ Average $\quad$ Square deviation

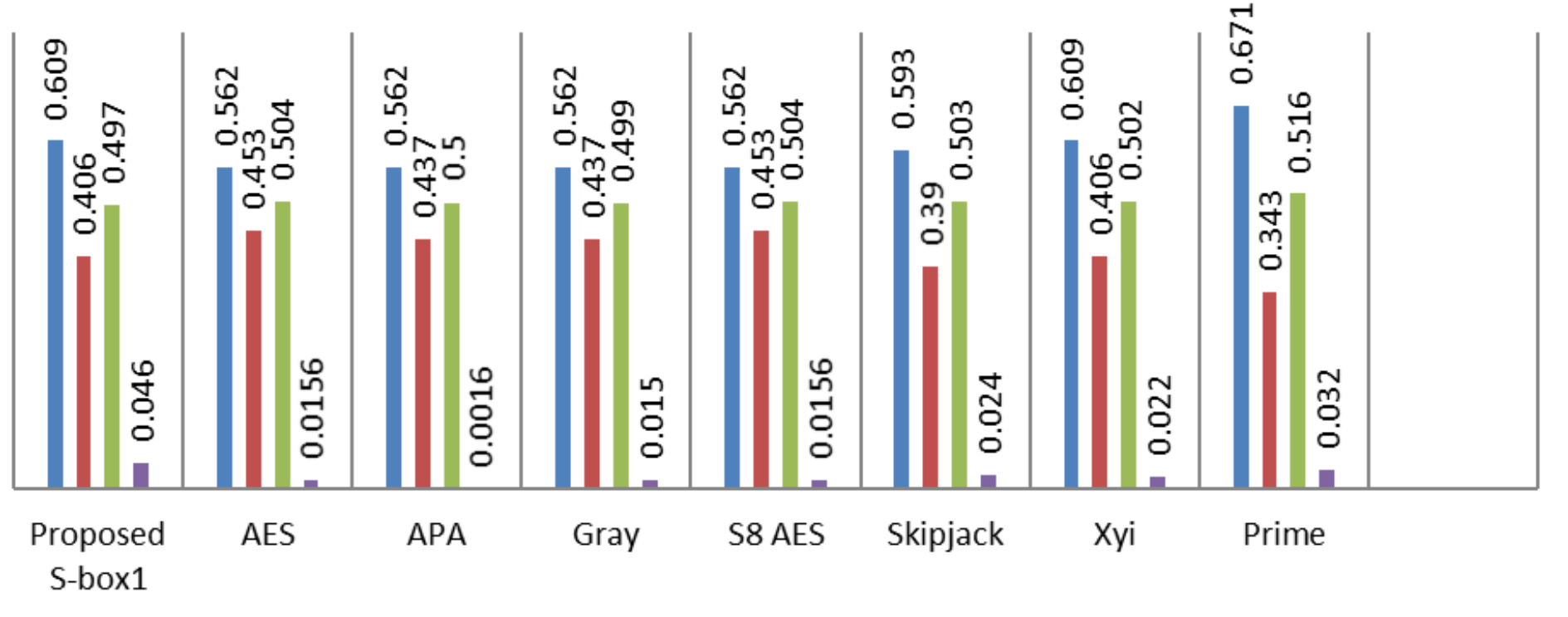

Figure 4

Strict Avalanche criterions

\section{Bic analysis}

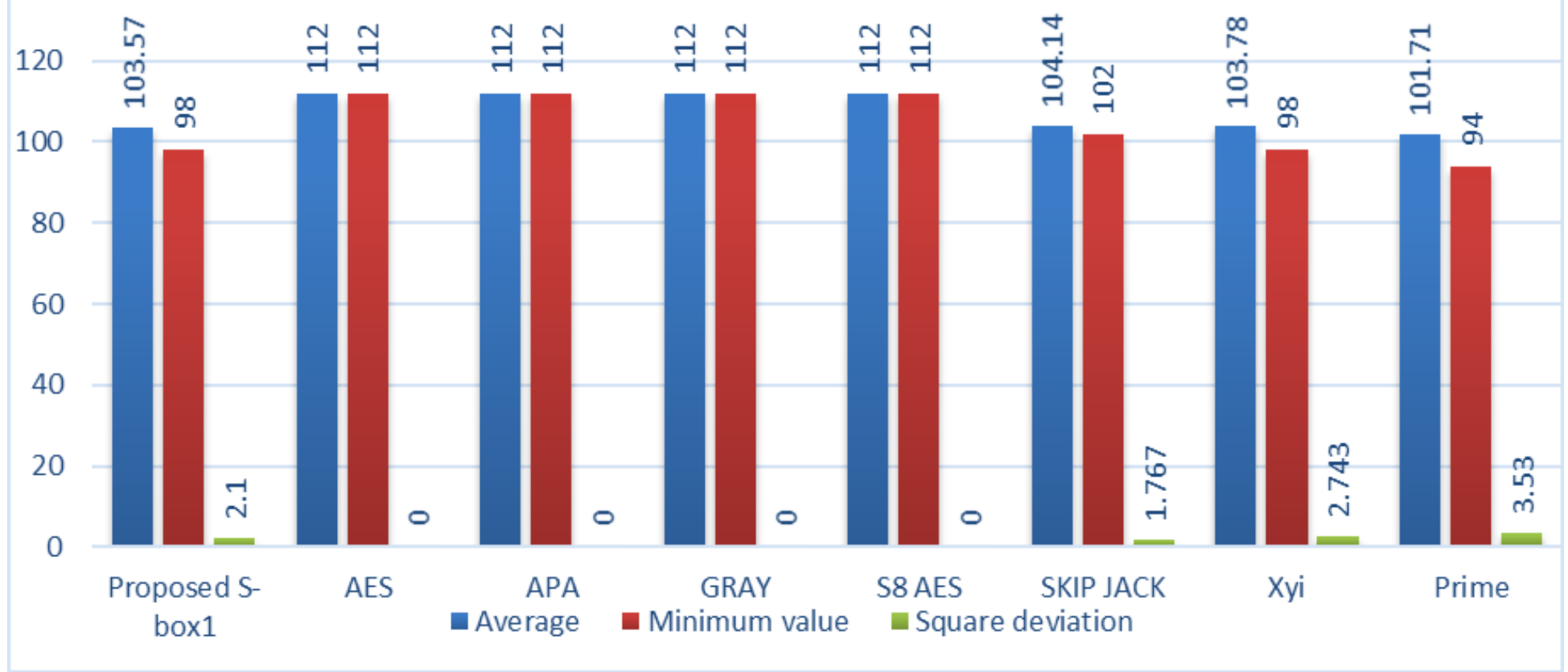

Figure 5

Bit Independence Criterion 


\section{Lp Analysis}

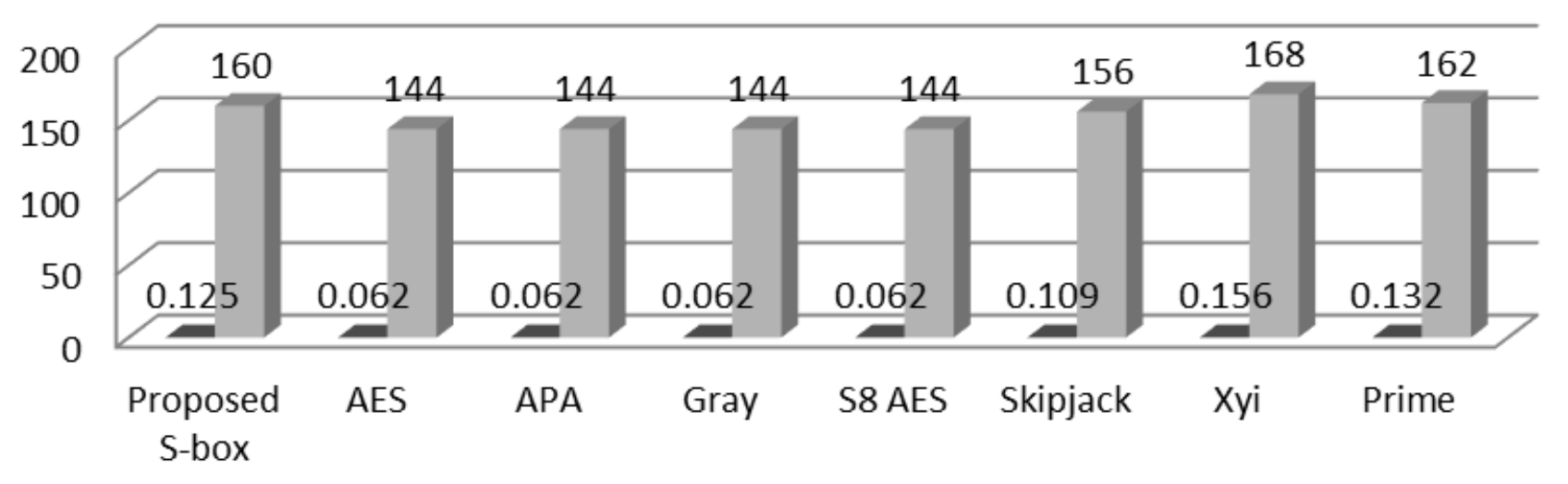

- Max Ip Max value

Figure 6

Linear Probability

\section{Dp Analysis of S-boxes}

$\square$ Maximum Dp
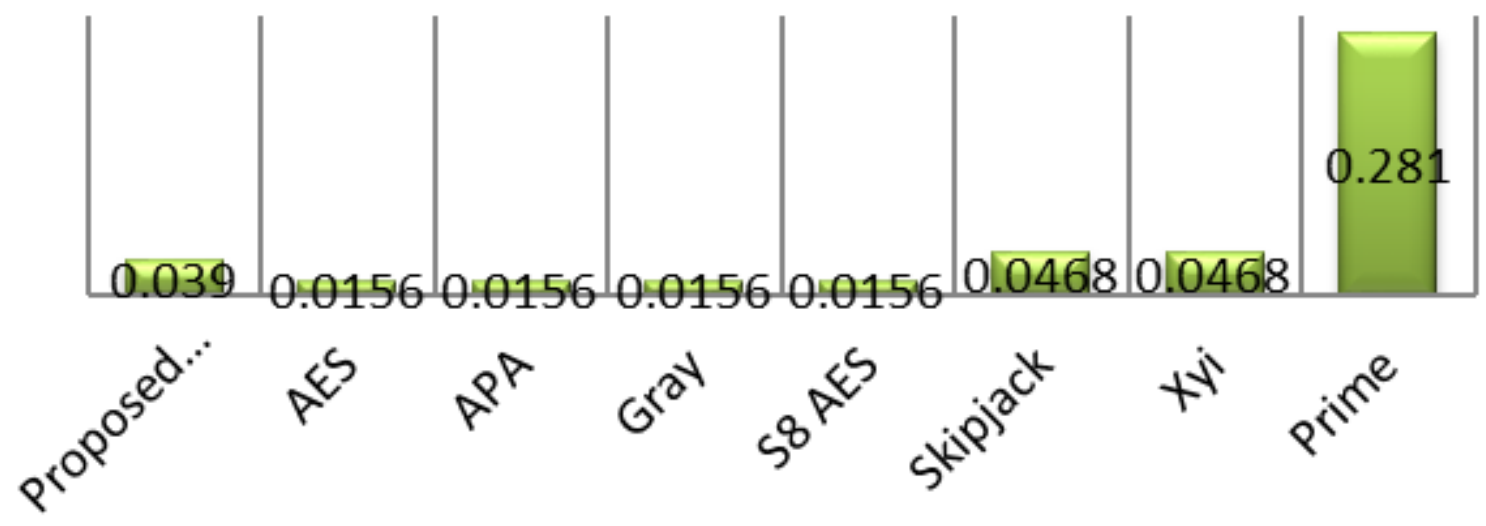

Figure 7

Differential probability 


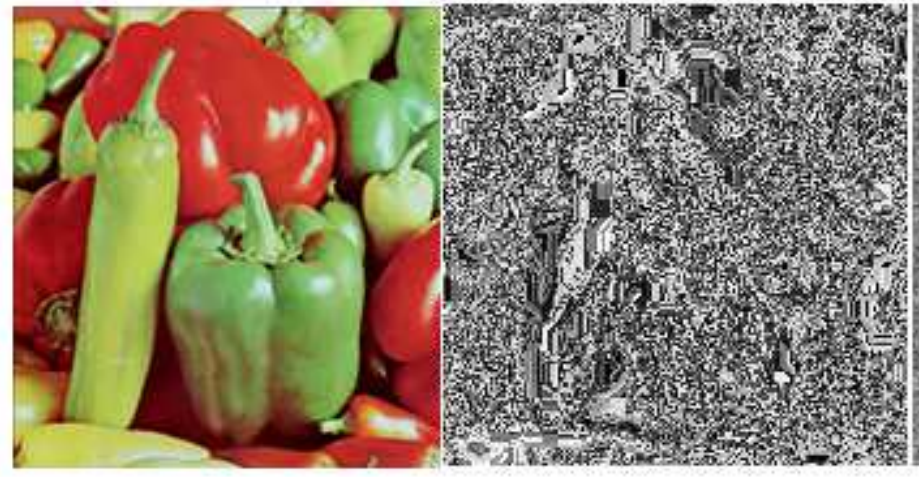

(a)

(b)

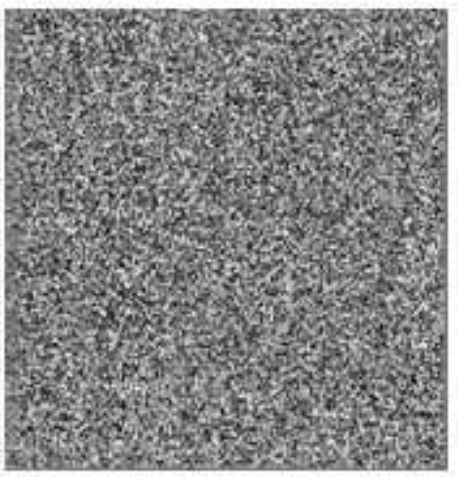

(c)

\section{Figure 8}

Capsicum Image Encryption (a) Original Image (b) First Round Encrypted Image and(c) Final Encrypted image 